\title{
Peptides as Drugs: Discovery and Development
}

\author{
Bernd Groner
}

"The necessity to exploit new drug targets and the suitability of peptides as drugs."

\section{1}

\section{Discovery of New Potential Drug Targets and the Limitations of Druggability}

Complex networks of interacting proteins constitute the signaling pathways which mediate the intracellular propagation of biological information. Signals can originate from the cell surface and be relayed to sites in the cell where a biological response is triggered. The recognition of receptors by specific ligands is usually the initiating event which regulates cellular homeostasis, but also causes cellular responses such as proliferation, migration, angiogenesis, immune responses and cell death. The transient assembly of higher-order protein complexes, mediated by specific protein interaction domains and often regulated by secondary protein modifications, underlies the signaling mechanisms. In many instances platform proteins, for example, bring together enzymes and substrates and, in turn, recruit negative regulators which assure the transient nature of the signaling processes. The high complexity of these interactions makes them susceptible to disturbances arising from mutations in participating components, and the deregulation of specific protein-protein interactions has been recognized as the cause of diverse diseases. Conversely, the aberrant interaction of proteins is often a hallmark of diseased cells, and the inhibition of interactions required either to initiate or to maintain a particular disease state provides challenging opportunities for targeted therapies.

The importance of specific protein interactions has not only been recognized for diseases which originate from mutations in endogenous genes, but also extends to exogenous causes of disease and pathogenic microorganisms. Today, many disease-causing organisms and diseases are starting to be understood in molecular detail [1], with almost 600 microbial genomes having already been sequenced and 1800 others currently under investigation. This has led to the 
identification of virulence genes, metabolic pathways and cell-surface proteins as new targets for antimicrobial drug development and candidate vaccines. New directions are primarily set by technologies aimed at the elucidation of global gene expression patterns, and these high-throughput molecular profiling techniques have accelerated the discovery of drug targets. Genomics, transcriptomics and proteomics not only play a decisive role in the investigation of infectious diseases, but also are becoming increasingly important in the understanding of multigenic human diseases such as diabetes, heart diseases and cancer [2]. However, the task to integrate such global and descriptive analyses into manageable models has only just begun, and the large and unwieldy datasets available not only still preclude the rational prediction of gene functions in an organismal context, but also hamper predictions about the benefits and side effects of targeted drugs.

The present limitations concerning the evaluation and interpretation of datasets collected from global gene expression patterns are not deterring progress, however. Today, large-scale efforts are under way to gain insights into whole genome alterations that distinguish cancer cells from their normal counterparts [3, 4]. Cancer cells exhibit multiple genetic alterations in their DNA sequences, in the number of individual genes, and in their epigenetic DNA and histone modifications. These alterations cause both the activation and inhibition of biological events, interpretable in the context of the pathophysiology of cancer cells [5]. The comparison of the human genome which is present in normal, healthy cells with that present in breast, colon, pancreatic cancer cells and glioblastomas, has shown that hundreds of genes can be present in mutated forms. Although about 60 genes have been found to be altered in individual tumors, the mutations varied when individual tumors were compared. Initially, it seems difficult to distinguish molecular alterations which are causal and drive tumor-related phenotypes, such as cell proliferation, cell death, metabolism, metastasis, angiogenesis or immune evasion, and those which are correlative and do not contribute to tumor formation. Nevertheless, consistent patterns could be identified. The most important mutations affect a limited number of cellular signaling pathways. The suggestion is that, interference with these pathways-but not necessarily the targeting of mutated gene products - might represent the most promising approach to therapy [3].

Elucidation of the functions of many of mutated gene products supports the overriding importance of deregulated pathways. Both, oncogenes and tumor suppressor genes control crucial points in the cell cycle, transitions from a resting stage $\left(G_{0}\right.$ or $\left.G_{1}\right)$ to a replicating phase $(S)$, inhibit cell growth, and stimulate cell death when induced by cellular stress [6]. The cells continuously respond to 'prods' emanating from external and internal signals. The oncogene and tumor suppressor gene products are usually components of signaling cascades and are integrated in networks of protein interactions. These protein interactions are most frequently regulated through post-translational modifications [7]. Proteins such as histones, p53, RNA polymerase II, tubulin, Cdc25C and tyrosine kinases can be modified at multiple sites through phosphorylation, acetylation, methylation, ubiquitination, sumoylation, and citrullination. These modifications can act in a com- 
binatorial fashion and constitute regulatory programs. Covalent modifications can modulate protein interaction domains and coordinate intermolecular and intramolecular signaling.

The multitude of molecules functionally involved in cancer formation and progression provides a rich source of potential points of interference, although the exploitation of these possibilities is still in its initial stages. The heterogeneity of tumor cells, mirrored in the large variety of mutations found, makes it difficult to define the most promising drug targets. Such targets should comprise molecules which are functionally crucial for cancer cells, and at least temporarily dispensable for normal cells. As the mutations found in tumor cells are not random, and the functional consequences are usually manifested in deregulated signaling pathways, the targeted inhibition of components encoded by oncogenes or components activated by oncogene products, has attracted much attention. Tumor cells do encode proteins, on which they are totally dependent-that is, the downregulation or functional inhibition of such components causes tumor cell death. The inactivation of such a component in normal cells is often tolerated and has led to the concept of "tumor cell oncogene addiction" [8]. Such proteins appear as appealing drug targets, and the development of inhibitors of oncogenic kinases-such as members of the EGF receptor family, BCR-ABL, PDGF receptor or c-KIT-has led to most impressive clinical advances. Other kinase inhibitors have been approved or are currently under development $[9,10]$. Since components of oncogenic pathways, however, are not always necessarily kinases, pharmacological problems must also be addressed.

The theoretical considerations of pharmacologists were applied to information gained from sequencing of the human genome, and resulted in an estimate of a relatively small number of molecular drug targets [11]. The term "druggable genome" was coined with the intent to delimit the subset of molecular targets for which orally available, commercial drugs could be developed. The majority of currently used drugs are directed towards classical druggable targets such as enzymes, G-protein coupled receptors (GPCRs), carriers and nuclear hormone receptors (NHRs) and ion channels [12]. The term "druggable" refers to targets which exhibit protein folds capable of interacting with drug-like chemical compounds. These compounds recognize the substrate-binding pockets of proteins, and it is for this reason that enzymes have mainly been used as therapeutic drug targets. Typically, they provided the prerequisites to develop rapid, sensitive screening assays and the detection of low-molecular-weight inhibitors that blocked the active site. Many of the inhibitors are derivatives of substrates-analogues which serve as starting points for the development of specific drugs. Such compounds are then further modified to exhibit a defined set of properties which lend them satisfactory pharmacokinetics [13]. However, in the case of proteins which interact with other proteins-rather than with small substrate molecules-a lack of binding pockets means that this approach is not generally applicable. Proteins which do not exhibit structural features that allow the derivation of such small-molecular-weight binders are considered by pharmacologists as unlikely to be modulated in their function through pharmaceutical intervention [14, 15]. 
Since many of the molecules that play crucial roles in disease processes as diverse as infectious diseases, diabetes, heart diseases and cancer cannot easily be modulated by small-molecular-weight inhibitors which recognize particular binding pockets, it is becoming mandatory to design and exploit molecules that do not necessarily fit the description of classical drug classes. Biological molecules and structures-such as peptides, recombinant proteins, antibodies, therapeutic genes or even whole cells-have the potential to fill part of this gap and thus blur the current distinction between "druggable" and "nondruggable" target molecules.

\section{2}

\section{Protein Interaction Domains Are at the Core of Signaling Pathways}

Modular interactions among proteins are at the heart of protein functions and cellular organization. Biological signaling requires that protein complexes are formed and activated at the right time and in the right place, and that their formation is both reversible and transient. The strength and duration of a signal may be critical for the effects of hormones, cytokines and growth factors, and a large number of specific protein interaction domains are known which mediate this machinery [16]. Since many proteins thus exert their biological activity through interactions with other proteins, an interference with such interactions would represent an attractive option. Moreover, those molecules capable of preventing these interactions could become a new class of drugs.

The structural diversity of the possible protein interfaces involved pose chances and challenges [17]. The chances are presented by the diversity of the interactions and the possibility for subtle perturbations. However, there exist also a number of potential problems, stemming from the dimensions of the targeted structures and from the relative inexperience in dealing with them. The main features of these difficulties have recently been identified [18], and range from structural to cell biological aspects. Protein interfaces and their interactions are not easily inhibited by low-molecular-weight compounds, and the search for specific inhibitors might therefore require new strategies. Intracellular protein concentrations are very high, estimated at $100 \mathrm{mg} \mathrm{ml}^{-1}$, while protein complexes may comprise identical subunits or different subunits. Oligomers can be formed in conjunction with protein synthesis, and result in so-called "obligate complexes"; alternatively, later on in the life of the protein they may form nonobligate complexes. Protein complexes exhibit different stabilities, and can be regulated through secondary modifications of the participating components [7]. In addition, complex formation and stability can be modulated by effector molecules or changes in their relative ratios and localization.

Protein complexes are held together by their contact interfaces. The size of the subunit interfaces is large, and ranges from approximately 300 to $4800 \AA^{2}$ [19]. The structure of participating proteins can undergo conformational changes when they form large interfaces [20]. Recognition patches are essential for binding, and 
usually one per interface has been found. Therefore, the design of inhibitors of protein-protein interactions, especially with small organic molecules, is a difficult and challenging-but not impossible-task. The principal of selective interference of protein-protein interactions with small synthetic molecules is actively being explored [21, 22]. Recently, transcription factors have received attention as suitable target structures, because they can be considered as end points of signal transduction pathways, and many of them also require dimerization in order to assume DNA binding activities. The dimerization domains and the DNA-binding domains of transcription factors, such as the hypoxia-inducible factor (HIF)-1, c-Myc and signal transducer and activator of transcription (STAT) 3, are currently being investigated, while drug-like small molecules are being sought which might potently and selectively inhibit these functional domains.

\section{3}

\section{Peptides as Inhibitors of Protein Interactions}

Proteins and peptides offer alternatives as interfering agents when the inhibition of protein-protein interactions is being considered. If protein interfaces mediate the contact between two proteins, then a compound mimicking the properties of one of the interfaces should act as a competitive inhibitor and prevent interaction of the binding partners. Peptides derived from such protein-protein interaction sites could possibly serve as antagonists. Proteins usually interact through interfaces which comprise proline, isoleucine, tyrosine, tryptophan, asparagine and arginine residues, and using this information peptides have been identified which act as agonists or antagonists of, for example, cell-surface receptors [23].

The use of peptides as specific inhibitors of protein-protein interactions might circumvent a number of problems which have been associated with smallmolecular-weight compounds considered for the same purpose. Although, the large surface areas involved in protein-protein interactions may be dissected into much smaller contact points, and only a small number of amino acids might actually be critical for the specificity of the interactions, the general rules are still difficult to derive [24]. A straightforward approach for the discovery of peptides which might serve as potential antagonists is an exploitation of the complementary surfaces of naturally occurring binding partners. These peptides could function as competitive inhibitors, masking an interaction site and making it inaccessible for the binding of the protein from which it has been derived. These peptides themselves could serve as potential drugs, if problems such as affinity, stability, delivery and specificity can be managed. Alternatively, information about the structure of the crucial amino acids that constitute the contact site could be used as a basis to design mimetics; indeed, this strategy has been used to develop peptide ligand mimetics for integrin receptors [25].

The vast complexity of random peptide libraries provides another possibility to identify specific peptide ligands. Peptides can be selected to fit any macromolecular surface and thus may act for example as ligands for functional domains of 
proteins. If the functional domain is appropriately chosen, the peptide aptamers may bind to protein targets and be able to interfere with the target function. Although the mechanism of action can be based on the masking of a binding domain for an interaction partner, it can also result from conformational changes induced as a consequence of the ligand-target interaction. Peptide aptamers are comprised of a variable peptide region of 8 to 20 amino acids in length, displayed in a scaffold protein, and have been selected for multiple targets. The isolation and use of peptide aptamers as inhibitors of individual signaling components represents an excellent starting point for highly selective and unconventional drugs, and also a new challenge for drug development [26-28].

The use of proteins and peptides as competitive inhibitors for any functional domain of a given target protein is most attractive, and would increase the subtlety of targeted interference considerably. Despite the conceptual appeal to develop protein antagonists as therapeutic agents, there are many technical and biological hurdles which must first be overcome. The affinity between a peptide ligand and a target domain can depend on multiple parameters, and may need to be optimized by mutational analysis and array procedures. The form in which a proteinprotein interaction is integrated into a particular high-molecular-weight complex in vivo is difficult to predict. The complexity of a multiprotein assembly, such as a ribosome or a transcription complex, entails a large number of interactions, and the consequences of a single interruption could be compensated by other interactions. Kinetic parameters, the intracellular localization and accessibility of the target structure, and the immunogenicity and stability of the construct into which the peptide is integrated, are all determinants of the potential therapeutic usefulness.

The discovery and development of peptide-based drugs have both rational and empirical aspects. Random screening procedures can be used to identify ligands for known functional domains of target proteins in high-complexity libraries. These screens can be complemented by structural, computational or biochemical information to identify ligands for targets for which natural interaction partners are known. Biotechnology will then allow the supply of such ligands with the additional functional domains that are required if they are to be used as drugs. Integration into scaffold proteins, the provision of a protein transduction domain, as well as favorable properties for recombinant expression and protein purification, are all required if the peptide itself is to be used as an effector. Ironically, many of these issues are reminiscent of the "rocky road" which monoclonal antibodies had to take before they became a commercial success.

However, the development of peptides into tools for diagnostic purposes and drugs, based on their fantastic specificity of target recognition and their versatility of mechanisms by which they can exert interference with protein functions, offers enormous promise. While peptides as drugs is a concept that still involves considerable challenge, encouragement may be gleaned from the wise words of the visionary Arthur C. Clarke (1917-2008), who admitted that “...we do not have all the answers, but we have plenty of questions certainly worth thinking about; and when the technology is finally sufficiently advanced it is indistinguishable from magic". 


\section{References}

1 Kaushik, D.K. and Sehgal, D. (2008) Developing antibacterial vaccines in genomics and proteomics era. Scand. J. Immunol., 67 (6), 544-52.

2 Chen, Y., Zhu, J. et al. (2008) Variations in DNA elucidate molecular networks that cause disease. Nature, 452 (7186), 429-35.

3 Jones, S., Zhang, X., Parsons, D.W. et al. (2008) Core signaling pathways in human pancreatic cancers revealed by global genomic analyses. Science, 321 (5897), 1801-6.

4 Parsons, D.W., Jones, S., Zhang, X. et al. (2008) An integrated genomic analysis of human glioblastoma multiforme. Science, 321 (5897), 1807-12.

5 Greenman, C., Stephens, P. et al. (2007) Patterns of somatic mutation in human cancer genomes. Nature, 446 (7132), 153-8.

6 Vogelstein, B. and Kinzler, K.W. (2004) Cancer genes and the pathways they control. Nat. Med., 10 (8), 789-99.

7 Seet, B.T., Dikic, I. et al. (2006) Reading protein modifications with interaction domains. Nat. Rev. Mol. Cell. Biol., 7 (7), 473-83.

8 Sharma, S.V. and Settleman, J. (2007) Oncogene addiction: setting the stage for molecularly targeted cancer therapy. Genes Dev., 21 (24), 3214-31.

9 Collins, I. and Workman, P. (2006) New approaches to molecular cancer therapeutics. Nat. Chem. Biol., 2 (12), 689-700.

10 Sharma, S.V., Bell, D.W. et al. (2007) Epidermal growth factor receptor mutations in lung cancer. Nat. Rev. Cancer, 7 (3), 169-81.

11 Hopkins, A.L. and Groom, C.R. (2002) The druggable genome. Nat. Rev. Drug Discov., 1 (9), 727-30.

12 Betz, U.A., Farquhar, R. et al. (2005) Genomics: success or failure to deliver drug targets? Curr. Opin. Chem. Biol., 9 (4), 387-91.

13 Keller, T.H., Pichota, A. et al. (2006) A practical view of 'druggability'. Curr. Opin. Chem. Biol., 10 (4), 357-61.
14 Imming, P., Sinning, C. et al. (2006) Drugs, their targets and the nature and number of drug targets. Nat. Rev. Drug Discov., 5 (10), 821-34.

15 Russ, A.P. and Lampel, S. (2005) The druggable genome: an update. Drug. Discov. Today, 10 (23-24), 1607-10.

16 Pawson, T. (2004) Specificity in signal transduction: from phosphotyrosine-SH2 domain interactions to complex cellular systems. Cell, 116 (2), 191-203.

17 Li, S., Armstrong, C.M. et al. (2004) A map of the interactome network of the metazoan C.elegans. Science, 303 (5657), 540-3.

18 Chene, P. (2006) Drugs targeting protein-protein interactions. ChemMedChem, 1 (4), 400-11.

19 Pal, A., Chakrabarti, P. et al. (2007) Peptide segments in protein-protein interfaces. J. Biosci., 32 (1), 101-11.

20 Wodak, S.J. and Janin, J. (2002) Structural basis of macromolecular recognition. $A d v$. Protein Chem., 61, 9-73.

21 Berg, T. (2008) Inhibition of transcription factors with small organic molecules. Curr. Opin. Chem. Biol., 12 (4), 464-71.

22 White, A.W., Westwell, A.D. et al. (2008) Protein-protein interactions as targets for small-molecule therapeutics in cancer. Expert. Rev. Mol. Med., 10, e8.

23 Jones, D.S., Silverman, A.P. et al. (2008) Developing therapeutic proteins by engineering ligand-receptor interactions. Trends Biotechnol., 26 (9), 498-505.

24 Lo Conte, L., Chothia, C. et al. (1999) The atomic structure of protein-protein recognition sites. J. Mol. Biol., 285 (5), 2177-98.

25 Sillerud, L.O. and Larson, R.S. (2005) Design and structure of peptide and peptidomimetic antagonists of proteinprotein interaction. Curr. Protein Pept. Sci., 6 (2), 151-69.

26 Baines, I.C. and Colas, P. (2006) Peptide aptamers as guides for small-molecule drug discovery. Drug Discov. Today, 11 (7-8), 334-41. 
27 Borghouts, C., Kunz, C. et al. (2005) Current strategies for the development of peptide-based anti-cancer therapeutics. J. Pept. Sci., 11 (11), 713-26.
28 Borghouts, C., Kunz, C. et al. (2008) Peptide aptamer libraries. Comb. Chem. High Throughput Screen., 11 (2), 135-45. 